\title{
Repercussões do diagnóstico de câncer de mama feminino para diferentes faixas etárias
}

\author{
Repercussions of the diagnosis of female breast cancer in different age groups
}

Humberto Moraes Silva Furtadoa, Sávio Campos Rodriguesa, Cintia Bragheto Ferreirab ${ }^{b}$ Taiane Freitas Lima ${ }^{a}$

a Psicólogo graduado pela Universidade Federal de Goiás (UFG), Regional Jataí.

b Psicóloga. Doutora em Enfermagem em Saúde Pública pela Escola de Enfermagem de Ribeirão Preto da Universidade de São Paulo (USP).

Professora Adjunta do Curso de Psicologia da UFG, Regional Jataí.

RESUMO

Objetivo: Identificar e descrever as repercussões do diagnóstico de câncer de mama para mulheres jovens abaixo dos 50 anos e acima dos 50 anos, residentes em um município goiano, na tentativa de compreender suas similaridades e diferenças.

Materiais e Métodos: Pesquisa qualitativa, com entrevistas semiestruturadas, que envolveu três mulheres jovens e três mulheres idosas com a confirmação do diagnóstico. A análise das entrevistas utilizou a perspectiva construcionista social, o que resultou na elaboração das seguintes temáticas: ser mulher; o diagnóstico; repercussões do tratamento. Resultados: Os resultados mostraram que em ambos os grupos, com exceção de uma mulher idosa, o diagnóstico de câncer promoveu reflexões relacionadas à feminilidade e um sofrimento intenso, que foi minimizado na relação das entrevistadas com a religiosidade.

Conclusão: O sofrimento intenso apresentado pela maioria das entrevistadas, na vivência do câncer, demonstra a necessidade de uma assistência em saúde potente para a ampliação das estratégias de enfrentamento de mulheres com câncer de mama.

Palavras-chave: neoplasias da mama; diagnóstico; feminilidade.

Objectives: To identify and describe the impact of the diagnosis of breast cancer in young women below 50 years old and older women over 50 years old living in the county of Goiás, to understand the similarities and differences.

Materials and Methods: A qualitative research was carried out through semi-structured interviews with three young women and three older women who had received a breast cancer diagnosis. The interviews were analyzed through a social constructionist perspective, which resulted in the following topics: being a woman; breast cancer diagnosis, and the impact of treatment.

Results: The results showed that in both groups, except for an elderly woman, the diagnosis provokes reflections related to femininity and intense suffering, which were reduced to a religious experience.

Conclusion: The intense suffering presented by most of the women interviewed, regarding the cancer experience, demonstrates the need for a powerful health care assistance in order to expand coping strategies for women with breast cancer.

Keywords: breast neoplasms; diagnosis; femininity. 


\section{INTRODUÇÃO}

Segundo o Instituto Nacional do Câncer, o câncer de mama é a neoplasia que ocorre com maior frequência em indivíduos do sexo feminino e representa o tipo de câncer mais encontrado no mundo. O câncer de mama é menos frequente antes dos 50 anos, porém após essa idade o número de novos casos é mais frequente. Dados estatísticos apontam para um aumento de novos casos em uma escala global, atingindo tanto países desenvolvidos como países em desenvolvimento. Para 2016, o INCA apresenta uma estimativa de 57.960 novos casos de câncer de mama no Brasil. As taxas de mortalidade por câncer de mama no Brasil permanecem altas, possivelmente devido à falta de diagnóstico em um estadio inicial da doença, mas se o câncer for diagnosticado e tratado precocemente, pode-se obter um bom prognóstico ${ }^{1}$.

Historicamente o câncer é uma doença que foi representada como uma das piores calamidades públicas do mundo, tendo sua história carregada de representações constrangedoras. Uma dessas representações, advindas das três primeiras décadas do século XX, fazia uma associação entre o câncer e a falta de higiene da alma e do corpo. Enfrentar o câncer tinha o significado de redenção, principalmente no caso da mulher, era chance de se redimir de ações que na época eram repreendidas socialmente, como comportamentos anti-higiênicos e mulheres que exerciam a sexualidade demasiadamente, implicando assim em um ato heroico lidar com o sofrimento até a ocasião da morte. O câncer foi associado ainda à imagem de uma doença letal, inglória, designada aos imorais² ${ }^{2}$, mística e invasiva $^{3,4}$, resultado de uma maldição ${ }^{5}$. Igualmente foi associado também a problemas irresolutíveis, assim como a corrupção e a violência ${ }^{6}$.

É comum que hajam em mulheres acometidas por câncer de mama sintomas depressivos. Estes podem ser atribuídos ao tratamento quimioterápico, assim como às contínuas dores causadas pela doença ${ }^{7}$. Muitas mulheres significam o câncer de mama como algo atemorizante, destrutivo e triste, constituindo-se, dessa maneira, em uma ameaça à saúde física e mental ${ }^{8}$. O paciente com câncer geralmente é vítima de um olhar diferenciado das pessoas próximas, que tendem a tratar o enfermo como alguém que tem pouco tempo de vida. Ao invés de buscar a motivação do paciente, dando suporte emocional para a continuidade do tratamento, as pessoas a sua volta se interessam por questões como procurar saber o tempo restante de vida do paciente, e o indivíduo com câncer consegue compreender quando as pessoas ao seu redor lhe tratam de forma piedosa, pois, mesmo disfarçando, os sentimentos manifestam-se ${ }^{9}$.
As mudanças psicológicas resultantes da descoberta do câncer de mama tem início quando a mulher começa a suspeitar, a partir do autoexame, que o nódulo encontrado possa ser um câncer ${ }^{9}$. Frente à doença, a mulher experimenta alterações em suas relações interpessoais e com ela própria. Essa mulher demanda não só auxílio médico-hospitalar, mas também um auxílio humanizado, que focalize a pessoa como alguém que está sofrendo, entretanto, que permanece com sua essência. Após o diagnóstico acontecem mudanças no dia-a-dia da mulher acometida por câncer de mama e seria importante que essas mudanças não fossem ignoradas, pois é nesse momento que se inicia uma fase decisiva, em que é necessário o recebimento de informações relacionadas à organização de sua vida ${ }^{10}$. Informações tais como a escolha de um acompanhante da confiança da paciente e a importância de buscar participar das decisões do seu tratamento junto aos profissionais de saúde, ou seja, a identificação e a potencialização de estratégias de enfrentamento positivas.

A mulher pode apresentar maior fragilidade psicológica frente à maior probabilidade da morte. Essa fragilidade é ainda mais marcante no câncer de mama, pois este pode provocar mutilações físicas, que, consequentemente, poderão transformar a aparência física, levando, muitas vezes, a sentimentos depressivos, ansiosos e temor frente à possibilidade do fim da vida. Isso pode acarretar também falta de confiança nos tratamentos e uma provável batalha pela sobrevivência ${ }^{10}$. Muitas vezes, o diagnóstico de câncer também pode promover um efeito avassalador na vida dos indivíduos que o recebem. Os motivos podem variar desde as perdas nos campos material, emocional e social, além de um grande desgaste físico e emocional decorrentes do período do tratamento e do próprio medo da morte. Devido a este quadro é indispensável que o paciente oncológico receba auxílio e atenção psicológica em seu tratamento ${ }^{11}$. Por isso, para que uma mulher possa superar as restrições da doença é necessário que ela construa novas expectativas de vida: reafirmando sua identidade dentro de uma nova compreensão de si mesma, formando dessa maneira, um novo "eu", possibilitando que haja por parte dessa mulher uma maior aceitação de sua condição enquanto portadora de câncer de mama ${ }^{10}$.

A compreensão da identidade feminina a partir da vivência do câncer de mama feminino pode ainda ser melhor compreendida não apenas analisando a trajetória histórica dos significados atribuídos ao câncer, mas também aos movimentos históricos e culturais ocorridos na década de 1960, que questionaram o lugar da mulher atrelado apenas ao cuidado do lar e dos filhos e que trouxeram um novo olhar sobre o lugar da mulher na família e na 
sociedade, marcado pelo ingresso no mercado de trabalho e na conquista de novos espaços, possibilitando a abertura para a liberdade de escolhas. Mas, ainda hoje, as mulheres vivem conflitos de paradigmas sobre o que é ser mulher e acumulam responsabilidades com as atividades domésticas e a educação e cuidado dos filhos ${ }^{12,13}$.

Tomando como referência os significados atribuídos ao câncer e ao ser mulher ao longo da história, realizamos um estudo anterior com mulheres do interior do estado de Goiás com faixa-etária inferior a 40 anos, com o intuito de compreender os sentimentos despertados nas entrevistadas a partir da descoberta do câncer de mama, e observamos que as participantes, ao se descobrirem com a doença, vivenciaram grande medo, atribuído principalmente à morte. As repercussões do diagnóstico de câncer de mama para mulheres jovens assemelham-se aos sentimentos das mulheres idosas com câncer de mama, os quais parecem estar associados a sofrimento, por acreditarem que o desfecho da doença será a morte. O câncer de mama desperta nessas mulheres surpresa e dor psíquica, significações que foram construídas durante a história da doença ${ }^{14,15}$. Todavia, apesar dos sentimentos negativos atribuídos ao câncer, as mulheres tendem a demonstrar fé na recuperação ${ }^{14} \mathrm{e}$ a relação com a religiosidade/espiritualidade como forma de enfrentamento do sofrimento experimentado ao se descobrirem com câncer ${ }^{14,16}$.

Contudo, diante da revisão da literatura realizada, identificou-se uma lacuna de pesquisas comparativas relacionando as repercussões do diagnóstico de câncer de mama, em mulheres de diferentes faixas etárias, residentes em um mesmo município brasileiro. A partir disso, observou-se a importância da realização de um estudo dessa natureza. Dessa maneira, tomando como referência a inexistência de estudos comparativos sobre a repercussão do diagnóstico de câncer de mama feminino entre mulheres jovens abaixo dos 50 anos de idade e mulheres acima dos 50 anos de idade ${ }^{1}$, assim como os significados históricos atribuídos ao câncer e ao ser mulher, o presente trabalho objetivou identificar e descrever as repercussões do diagnóstico de câncer de mama para esses dois grupos, residentes em um município goiano, na tentativa de compreender suas similaridades e diferenças.

\section{MATERIAIS E MÉTODOS}

O estudo realizado foi do tipo qualitativo, objetivando identificar e descrever as repercussões do diagnóstico de câncer de mama para mulheres jovens e idosas que o receberam, comparando os significados desse diagnóstico entre os dois grupos, propondo obter um olhar expandido do fenômeno em questão ${ }^{17}$.

\section{Instituição e Participantes}

O presente estudo teve como participantes pacientes do sexo feminino acometidas por câncer de mama, vinculadas a um Núcleo Regional de Combate ao Câncer, que é uma associação civil assistencial de direito privado, situada em um município goiano.

Dentre as mulheres cadastradas na referida instituição, foram selecionadas para a pesquisa as mulheres mais jovens e as mais velhas com câncer de mama, constituindo-se assim dois grupos. No primeiro grupo encontrou-se 3 mulheres abaixo dos 50 anos, constituído pela mais nova com 31 anos e a mais velha com 37 anos. No segundo grupo, também encontrou-se 3 mulheres, com idade superior aos 50 anos, sendo a mais nova com 56 anos e a mais velha com 71 anos. Portanto, 6 mulheres que haviam recebido o diagnóstico de câncer de mama compuseram a amostra do estudo.

\section{Procedimentos}

O estudo foi submetido e aprovado em um Comitê de Ética em Pesquisa (parecer no 120/13). Logo após a sua aprovação, o primeiro grupo de mulheres - constituído por mulheres jovens - foi convidado a participar da pesquisa. Os convites foram realizados por telefone, momento no qual também foram informados os objetivos do estudo. Em um momento anterior a cada uma das entrevistas foi realizada a leitura e assinatura do Termo de Consentimento Livre e Esclarecido. O mesmo procedimento foi aplicado às mulheres do segundo grupo, com faixa etária superior aos 50 anos.

Com cada uma das participantes foi feita uma entrevista do tipo semiestruturada. As entrevistas com o primeiro grupo de mulheres aconteceu dentro das dependências do Núcleo Regional, com a presença de uma pesquisadora e a entrevistada em questão. Com o segundo grupo de mulheres, as entrevistas foram realizadas na casa de cada uma das entrevistadas, com a presença de duas pesquisadoras e a entrevistada. As perguntas abordaram temáticas como os dados sociodemográficos das entrevistadas, o momento de recebimento da confirmação do diagnóstico de câncer de mama, o modo de lidar com a doença após a sua confirmação, bem como suas repercussões no cotidiano das pacientes.

Após a realização das entrevistas, as mesmas foram lidas exaustivamente para que os temas mais relevantes pudessem emergir das falas das participantes do estudo ${ }^{18}$. As temáticas, resultantes da leitura exaustiva das entrevistas, foram analisadas ancoradas na perspectiva construcionista social, a qual postula que os discursos são artefatos sociais produzidos coletivamente ao longo da história e da cultura ${ }^{19}$. A partir disso, a análise das referidas temáticas foi realizada 
ancorada nos significados do câncer, de suas repercussões na vida de mulheres que o experienciam, bem como do ser mulher, disponíveis na revisão apresentada neste estudo.

\section{RESULTADOS E DISCUSSÃO}

Inicialmente serão apresentadas as participantes dos grupos de mulheres jovens e idosas, respectivamente, assim como as temáticas que surgiram em suas falas, a saber: ser mulher; o diagnóstico e, repercussões do tratamento, associadas a uma comparação entre os discursos dos diferentes grupos referentes às temáticas citadas.

Conforme demonstrado na Tabela 1, com a caracterização das entrevistadas, no período das entrevistas, as participantes do grupo de mulheres jovens tinham em média 34 anos de idade. Dentre elas, uma participante concluiu o ensino médio e as outras interromperam os estudos na sétima série e terceiro ano. Duas delas tem dois filhos e uma participante tem 1 filho. Todas foram submetidas à cirurgia (duas participantes do tipo mastectomia radical e uma participante do tipo quadrantectomia com esvaziamento axilar). Todas as entrevistadas também foram submetidas a tratamentos complementares, como a quimioterapia e duas delas passaram por radioterapia.

A média de idade das mulheres mais velhas era de 62 anos. Uma das participantes concluiu o ensino médio e duas não possuem escolarização. Duas participantes tem três filhos cada e uma possui sete. Todas as participantes foram submetidas à cirurgia (mastectomia radical). Além disso, uma delas passou por quimioterapia e radioterapia, outra participante foi submetida à radioterapia e, até o momento da entrevista, a outra participante não havia sido submetida a nenhum tratamento complementar.

\section{Ser mulher}

A vivência do câncer de mama despertou nas participantes vivências relacionadas ao que é ser mulher. As entrevistadas do grupo de mulheres mais velhas, ao relatarem sobre o que é ser mulher, tiveram respostas que identificam a mulher como sinônimo de mãe e cuidadora do lar, reafirmando o lugar histórico atribuído à figura feminina como cuidadora da casa e dos filhos ${ }^{12,13}$.

"Ser mulher é bom. Porque a gente vai ter filhos da gente. Um esposo que dá valor na gente, né." (V1)

"Uai, eu acho assim que mulher nasceu pra arrumar a casa, né. Fazer as coisas, né?" (V2)

“(...) é ser mãe, tudo. Ser mãe, o importante é a gente ser mãe, dona de casa, mulher, companheira." (V3)

Contudo, com o ingresso da mulher no ensino escolarizado e saída para o mercado de trabalho, ela começa a se desprender da imagem unicamente pertencente as tarefas familiares, estabelecendo maior independência ${ }^{12,13}$. Esta afirmação pode ser encontrada no discurso de uma das mulheres jovens, ao relatar sobre o que é ser mulher.

"Ah, eu acredito que é ter a oportunidade de tá fazendo alguma coisa, trabalhar fora!" (N1)

Apesar de uma maior independência feminina ${ }^{12,13}$, os trabalhos domésticos e cuidados dos filhos e demais familiares continuam, ainda hoje, como as principais responsabilidades das mulheres ${ }^{20}$. Apesar de serem mulheres jovens, observase na fala de duas dessas participantes que o significado de ser mulher, ainda se assemelha ao significado dado pelas mulheres mais velhas.

Tabela 1. Caracterização da amostra.

\begin{tabular}{|c|c|c|c|c|c|c|}
\hline Participantes & Idade & Estado civil & Filhos (as) & Escolaridade & Profissão & Terapêuticas para o câncer \\
\hline N1 & 31 & Casada & 2 filhos & Médio completo & $\begin{array}{l}\text { Auxiliar serviços } \\
\text { gerais }\end{array}$ & $\begin{array}{l}\text { quimioterapia, mastectomia } \\
\text { radical e quimioterapia }\end{array}$ \\
\hline N2 & 37 & Viúva & 2 filhos & $\begin{array}{l}\text { Fundamental } \\
\text { incompleto }\end{array}$ & Do lar & $\begin{array}{l}\text { quimioterapia, } \\
\text { quadrantectomia com } \\
\text { esvaziamento axilar e } \\
\text { radioterapia }\end{array}$ \\
\hline N3 & 36 & Casada & 1 filha & Médio incompleto & Do lar & $\begin{array}{l}\text { quimioterapia, mastectomia } \\
\text { radical e radioterapia }\end{array}$ \\
\hline $\mathrm{V} 1$ & 71 & Divorciada & 3 filhos & Nunca estudou & Do lar & $\begin{array}{l}\text { mastectomia radical e } \\
\text { radioterapia }\end{array}$ \\
\hline V2 & 61 & Casada & 7 filhos & Nunca estudou & Do lar & $\begin{array}{l}\text { mastectomia radical, } \\
\text { radioterapia e quimioterapia }\end{array}$ \\
\hline V3 & 56 & Casada & 3 filhos & Médio completo & $\begin{array}{l}\text { Manicure e } \\
\text { vendedora }\end{array}$ & mastectomia radical \\
\hline
\end{tabular}


"Ser mãe é ser mulher." (N2)

"É muito bom, né, ser muié, cuidar da saúde, e agora ficou difícil, e muito, num posso lavar vasilha, num posso mais limpar a casa, num posso lavar roupa (...)." (N3)

Portanto, observou-se que ser mulher, na vivência do câncer de mama feminino, independentemente da faixa etária das participantes, emergiu muito mais associado aos valores tradicionais do que se espera de uma mulher, como o cuidado do lar, dos filhos e do marido, do que quando analisado em comparação à figura feminina de independência, pertencente aos valores modernos sobre o ser feminino ${ }^{12,13}$, presente em apenas uma das mulheres jovens entrevistadas.

\section{O diagnóstico}

Antes mesmo de receber o diagnóstico, a mulher começa a desconfiar de alterações físicas que surgem em seu corpo como, por exemplo, o descobrimento acidental de um nódulo que até o momento não havia sido descoberto. Por meio da descoberta dos primeiros sinais e sintomas, a mulher passa a desconfiar que algo não se encontra bem ${ }^{10}$, concordando com as falas das participantes apresentadas a seguir.

“(...) quando eu descobri, que eu tava, era bem pequenininho o nódulo, só que minha médica por duas vezes ela falou que não era grave (...). Na última vez que eu passei com a ginecologista, ela me falou que só ia acompanhar de três em três meses, eu que estranhei, pelo fato de estar muito grande (...)." (N1)

"Não fiquei nenhum pouquinho surpresa! Eu já tinha achado ele, né! Eu sentia que aquilo não estava certo mesmo." (V1)

"(...) eu que vi! Fiz um auto exame e tinha um modulozinho." (V3)

O câncer de mama provoca nas mulheres alterações físicas, psicológicas e sociais afetando-as fortemente. Ao experimentar o diagnóstico as mulheres vivem momentos de instabilidade emocional constantes, caracterizadas principalmente por sentimentos temorosos e incertos, em relação à doença ${ }^{10,16}$. A primeira reação observada nas mulheres diante do diagnóstico de câncer de mama é o de desespero, devido a ligação feita entre o câncer e a morte ${ }^{9,16}$.

Observa-se nas entrevistadas, quando perguntadas sobre os sentimentos frente ao diagnóstico, um discurso permeado de sentimentos relacionados ao medo da morte, bem como o sofrimento causado por esta confirmação. O medo da morte não é exclusividade das mulheres mais jovens entrevistadas.
As mulheres mais velhas também manifestaram sentimentos parecidos.

"Eu senti muito medo! Um pavor! Eu achava que ia morrer, eu só pensava nisso, eu achava que ia morrer, não tinha nada que me fizesse pensar o contrário (...)." (N1)

"Uma doença dessa quando cê recebe assim cê pensa logo que vai morrer (...). Eu fiquei sem chão (...)." (N2)

"Eu fiquei assim (...) Vou morrer! A primeira coisa que a gente pensa. No começo foi um choque." (N3)

"Minhas pernas acabou, me deu aquela tremedeira, danei a chorar, Deus me livre, nossa senhora. Mas como eu chorei." (V2)

"O dia que falaram pra mim mesmo parece que abriram o chão e eu entrei, não tem ninguém que não desespera, até hoje eu não vi ninguém que não desesperou." (V3)

Contudo, na fala de V1, apresentada a seguir, quando perguntada sobre o diagnóstico, percebe-se uma diferença em relação aos relatos anteriores que associaram o câncer a um choque e à morte. A referida entrevistada, pelo contrário, demonstrou tranquilidade e força, atribuídas ao mundo divino, concordando com estudos que apontam a religiosidade como uma estratégia de enfrentamento capaz de aliviar o sofrimento experimentado na vivência do câncer ${ }^{14,16}$.

"Não fiquei nenhum pouquinho surpresa! (...). Nunca derramei um pingo de lágrima por causa desse problema. De jeito nenhum! Até hoje, graças a Deus (...). Tô abençoada mesmo, sinto que eu tô curadíssima." (V1)

Tomando como referência as falas das participantes apresentadas, percebe-se que independentemente da faixa etária, as mulheres perceberam alterações em seus corpos anteriormente ao recebimento do diagnóstico que as fizeram vivenciar a doença antes mesmo de sua confirmação. Posteriormente a isso, a vivência do diagnóstico positivo foi vivenciado de forma negativa por ambos os grupos entrevistados, reafirmando o câncer como uma doença que desperta sofrimento intenso e o medo da morte ${ }^{9,16}$, com exceção de uma entrevistada, que vivenciou o diagnóstico com tranquilidade.

\section{Repercussões do tratamento}

O câncer de mama nos dias atuais é considerado como uma doença sistêmica, sendo assim, seu tratamento cirúrgico é acompanhado por terapias adjuvantes como a quimioterapia, que é um tratamento agressivo ${ }^{21}$. O tratamento 
quimioterápico provoca, nas mulheres acometidas por câncer de mama, sentimentos como ansiedade e medo, decorrentes de uma grande confusão a respeito do tratamento e suas limitações impostas, como mal-estar geral, náuseas e vômitos constantes ${ }^{9}$, os quais podem ser verificados nas falas apresentadas na sequência.

“(...) nossa, a quimioterapia me matava, eu fiz seis, e era toda semana." (V2)

"Eu tenho medo do tratamento, mais medo do que da doença, tem hora que só na primeira quimioterapia que eu fiz da vontade de desistir." (N1)

Dado o sentido estigmatizante, o câncer de mama faz com que a mulher passe por intenso sofrimento psíquico. Abafadas por esses sentimentos, as mulheres com câncer afastam-se de seu círculo social e familiar, por enfrentarem uma constante perda de identidade nessas relações ${ }^{10}$. Nas entrevistas, as mulheres de ambos grupos relataram grande sofrimento resultante da alteração em seus cotidianos, devido às perdas físicas decorrentes do tratamento.

“(...) eu fico mais triste não é por causa da doença, é pelo fato deu não poder fazer tudo que eu fazia antes (...) Como deu esse nódulo muito grande aqui (mostra no seio o local) e deu, eu acho que dois ou três aqui nas axilas, vai ter que tirar no nervo, aí provavelmente, eu seco cachorro banho o cachorro, então não vou conseguir fazer o movimento, pra mim a parte pior é isso" (N1).

“(...) faço terapia, dói demais, mas eu faço, esforço, eu quero andar de novo, eu quero trabalhar." (V3)

Apesar do discurso das mulheres relacionados ao tratamento estarem voltados aos sentimentos atemorizantes e ameaçadores despertados pelo câncer ${ }^{8}$, na fala de V1, apresentada a seguir, observa-se que ela não sentiu as complicações físicas como as demais mulheres.

"Nunca senti nada, dor nenhuma. Fiz a cirurgia, fiz oito sessão de química, fiz 25 radio, nunca senti nada. Graças a Deus!" (V1)

Todo o sofrimento experimentado pela maioria das entrevistadas na vivência do câncer, parece ter direcionado as participantes a uma aproximação com o universo divino, visto que a fé e/ou a religiosidade podem se apresentar como um recurso para a superação e aceitação da doença ${ }^{14,16}$, como é possível acompanhar na sequência.

"Deus tem abençoado, eu tenho muita fé em Deus. Tem muita gente mais fraca que eu, e eu vou passando." (N3)
“(..) mas a gente pensa muito em Deus. Deus é bom, né! Ele ajuda muito a gente. Se você tiver fé nele, ele ajuda a gente." (V1)

Dessa maneira, as repercussões do tratamento do câncer de mama, para ambos os grupos de entrevistadas, emergiu associado a um sofrimento intenso, com exceção de uma entrevistada. Porém, todo o sofrimento experimentado pelas participantes foi amenizado pela relação das participantes com o universo religioso ${ }^{14,16}$.

\section{CONSIDERAÇÕES FINAIS}

O estudo realizado demonstra que as mulheres jovens e as mulheres mais velhas tendem a vivenciar o diagnóstico de câncer de mama de formas muito semelhantes. Ambos os grupos, vivenciaram a enfermidade revivendo aspectos relacionados ao significado de ser mulher, assim como questões relacionadas ao próprio diagnóstico da doença e as repercussões do tratamento do câncer.

Ambos os grupos relataram os sentidos do que é ser mulher muito mais associado aos valores tradicionais do feminino, tais como associados aos cuidados dos outros e da casa, do que atrelados à independência feminina, os quais foram presentes em apenas uma das entrevistadas jovens. Além disso, o diagnóstico assim como as repercussões do tratamento também foram vivenciados pela maioria das entrevistadas com intenso sofrimento, com exceção de uma participante mais velha. Sendo que todo o sofrimento experimentado foi minimizado na relação das entrevistadas com o universo religioso.

Contudo, apesar dos dados do estudo se limitarem apenas a uma amostra de um município do interior brasileiro, não podendo portanto serem generalizados para todo o Brasil, parecem indicar que o câncer de mama ainda é uma doença que coloca para as mulheres que o vivenciam questionamentos relacionados à feminilidade, e que a despeito de campanhas educativas sobre a doença e suas possibilidades de cura, ainda é uma enfermidade carregada de sentidos negativos, como a morte. De outro lado, os dados apontam para a necessidade da ampliação de estudos dessa natureza, visto que ainda é incipiente no Brasil o conhecimento de como mulheres jovens vivenciam o câncer de mama e as possíveis relações dessas vivências com as mulheres mais velhas que também a vivenciam, o que poderá auxiliar as equipes de saúde na construção de práticas de cuidado capazes de ampliar o repertório de possibilidades de enfrentamento dos pacientes que vivenciam o câncer de mama. 


\section{REFERÊNCIAS}

1. Brasil. Instituto Nacional do Câncer. Estimativa de incidência e mortalidade por câncer no Brasil [dados na Internet]. [acessado 2016 Jan 20]. Disponível em: http://www.inca.org.br

2. Sant'anna BD. A mulher e o câncer na história. In: Gimenez MGG, organizadora. A mulher e o câncer. Campinas: Psy; 1997. p. 43-70.

3. Aquino VV, Zago MMF. O significado das crenças religiosas para um grupo de pacientes oncológicos em reabilitação. Rev Latino-am Enfermagem. 2007;15(1):42-7.

4. Sontag S. Doença como metáfora, aids e suas metáforas. São Paulo: Companhia de Letras; 2007.

5. Brasil. Instituto Nacional do Câncer. Pesquisa de opinião pública dos brasileiros sobre o câncer [dados na Internet]. [acessado 2015 Mar 11]. Disponível em: http://www.inca.org.br

6. Gomes R, Skaba MMVF, Vieira RJS. Reinventando a vida: proposta para uma abordagem sócio-antropológica do câncer de mama feminina. Cad Saúde Pública. 2002;18(1):197-204. http://dx.doi. org/10.1590/S0102-311X2002000100020

7. Cangussu RO, Soares TBC, Barra AA, Nicolato R. Sintomas depressivos no câncer de mama: inventário de depressão de Beck - Short Form. J Bras Psiquiatr. 2010;59(2):106-10. http://dx.doi.org/10.1590/ S0047-20852010000200005

8. Fialho AVM, Silva RM. Câncer de mama: o pensar e o fazer das mulheres. Rev Bras Enferm. 2004;57(4):157-60.

9. Maluf MFM, Mori LJ, Barros ACSD. O impacto psicológico do câncer de mama. Rev Bras Cancerol 2005;51(2):149-54.

10. Araújo IMA, Fernandes AFC. O significado do diagnóstico do câncer de mama para a mulher. Esc Anna Nery. 2008;16(1):27-33. http:// dx.doi.org/10.1590/s1414-81452008000400009

11. Silva CR, Portella SDC, Reis LR, Bispo TCF. Religião e morte: qual a relação existente? Rev Enferm Contemp. 2012;1(1):130-41. http:// dx.doi.org/10.17267/2317-3378rec.v1i1.49
12. Magalhães IS. Entre a casa e o trabalho: a transmissão geracional do feminino [dissertação]. Rio de Janeiro (RJ): Pontifícia Universidade Católica do Rio de Janeiro; 2010.

13. Almeida LS. Mãe, cuidadora e trabalhadora: as múltiplas identidades de mães que trabalham. Rev do Dep Psicol UFF. 2007;19(2): 411-22. http://dx.doi.org/10.1590/S0104-80232007000200011

14. Gontijo IBR, Ferreira CB. Sentimentos de mulheres jovens frente ao diagnóstico de câncer de mama feminino. Ciênc Saúde. 2014;7(1):2-10. http://dx.doi.org/10.15448/1983-652X.2014.1. 15488

15. Silva LC. Câncer de mama e sofrimento psicológico: Aspectos relacionados ao feminino. Psicol Estud. 2008;13(2):231-7. http:// dx.doi.org/10.1590/S1413-73722008000200005

16. Ferreira CB, Almeida AM, Rasera EF. Sentidos do diagnóstico por câncer de mama feminino para casais que o vivenciaram. Interface. 2008;12(27):863-71. http://dx.doi.org/10.1590/S141432832008000400015

17. Godoy AS. Introdução à pesquisa qualitativa e suas possibilidades. Rev Adm Empres. 1995;35(2):57-63. http://dx.doi.org/10.1590/ S0034-75901995000200008

18. Spink MJP. Desvendando as teorias implícitas: uma metodologia de análise das representações sociais. In: Guareschi P, Jovchelovitch S, organizadores. Textos em representações sociais. Petrópolis: Vozes; 2000. p. 117-45

19. Gergen KJ. The social constructionist movement in modern psychology. Am Psychol. 1985;40(3):266-75. http://dx.doi. org/10.1037/0003-066X.40.3.266

20. Bruschini MCA. Trabalho e gênero no Brasil nos últimos dez anos. Cad Pesqui. 2007;37(132):537-72. http://dx.doi.org/10.1590/ S0100-15742007000300003

21. Makluf ASD, Dias RC, Barra AA. Avaliação da qualidade de vida em mulheres com câncer da mama. Rev Bras Cancerol 2006;52(1): 49-58. 